\title{
ON DEPOLARIZATION LIDAR-BASED METHOD FOR THE DETERMINATION OF LIQUID-CLOUD MICROPHYSICAL PROPERTIES
}

\author{
Gilles Roy $^{1^{*}}$, Xiaoying $\mathrm{Cao}^{2}$, Grégoire Tremblay ${ }^{3}$ and Robert Bernier ${ }^{4}$
}

${ }^{1}$ RDDC Valcartier 2459 de la Bravoure,Québec, Qc, Canada, gilles.roy@drdc-rddc.gc.ca

${ }^{2}$ Lidar Consultant, Ottawa, Canada; ${ }^{3}$ AEREX Avionic, Levis, Canada; ${ }^{4}$ IOSL, Mirabel, Canada,

\begin{abstract}
Under single scattering conditions, water droplets clouds do not depolarize the backscattered light. However, backscattered light from multiple scattering will be depolarized. The level of depolarization is a function of the droplets size, the cloud extinction coefficient value and profile; it has also an important dependency on the lidar field-of-view (FOV). The use of depolarization information to retrieve cloud microphysical properties, using Multiple-FOV has been the object of studies, [1], [2]. Recently the use of the depolarization, at a single FOV, has been studied for cloud with linear liquid water content profiles, [3], [4]. In this paper we present the mechanism leading to depolarization and identify the FOV values for which the information on particle size is high. Also Monte Carlo simulations for cloud with constant and ramp up profiles are presented. The degree of linear depolarization as a function of cloud penetration is significantly different for both cloud profiles. This suggests that the use of the degree of linear depolarization at a single FOV should be used with caution to determine clouds micro-physical parameters.
\end{abstract}

\section{DEPOLARIZATION RATIOS}

Backscattered light from spherical particles does not change the polarization orientation state of a linearly polarized incident light. This particularity allows the discrimination of spherical particles from non-spherical particles. However, under multiple scattering conditions, even spherical particles generate depolarized signals. The linear depolarization ratio $\left(\delta_{\text {lin }}\right)$ at a scattering angle $\beta$ is defined as the ratio of the power integrated over the azimuthal angles $\varphi$ in the plane of polarization orthogonal $\left(I_{\perp}\right)$ and parallel $\left(I_{/ /}\right)$to the linearly polarized source:

$$
\delta_{\text {lin }}(\beta)=\frac{\left.<I_{\perp}\right\rangle}{\left\langle I_{/ /}>\right.}=\frac{\int_{0}^{2 \pi} I_{y}(\beta, \varphi) d \varphi}{\int_{0}^{2 \pi} I_{x}(\beta, \varphi) d \varphi},
$$

Using Mie theory, it can be shown [1] that:

$$
\delta_{\text {lin }}(\beta)=\frac{\left(P_{2} \cos ^{2} \beta-2 P_{3} \cos \beta+P_{1}\right)}{\left(3 P_{2} \cos ^{2} \beta+2 P_{3} \cos \beta+3 P_{1}\right)}
$$

Where in $P_{1}, P_{2}$ and $P_{3}$ are the Mie scattering phase matrix elements defined by Deirmendjian.

Figures 1 shows the phase functions and the depolarization ratios at a wavelength of $0.532 \mu \mathrm{m}$ for linear incident waves on water-droplets of diameters $4 \mu \mathrm{m}$ and $20 \mu \mathrm{m}$. The water droplets size distribution is log-normal with a geometric standard deviation of 0.1 ; the distribution was truncated for values smaller than 0.02 the maximum of the distribution.

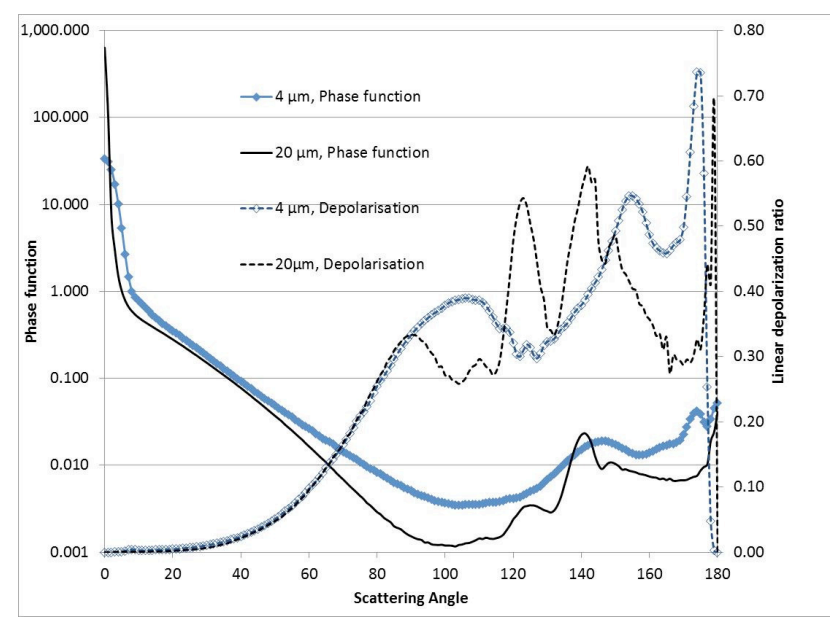

Figure 1. Phase functions and linear depolarization ratios for 4 and $20 \mu \mathrm{m}$ in diameter water droplets.

For depolarization, the major observations are:

- The light is not depolarized in the forward scattering direction for angles as large as $20^{\circ}$.

- The depolarization is equal to 0 at $180^{\circ}$.

- The depolarization increases very sharply around $180^{\circ}$.

Figure 2 shows the depolarization as a function of scattering angles ranging from $170^{\circ}$ to $180^{\circ}$ for 
water droplets of $4,8,12,16$ and $20 \mu \mathrm{m}$ (same distribution as for figure 1). For smaller particles, the depolarization peak shifts toward smaller scattering angles. However the maximum values keep very close to each other.

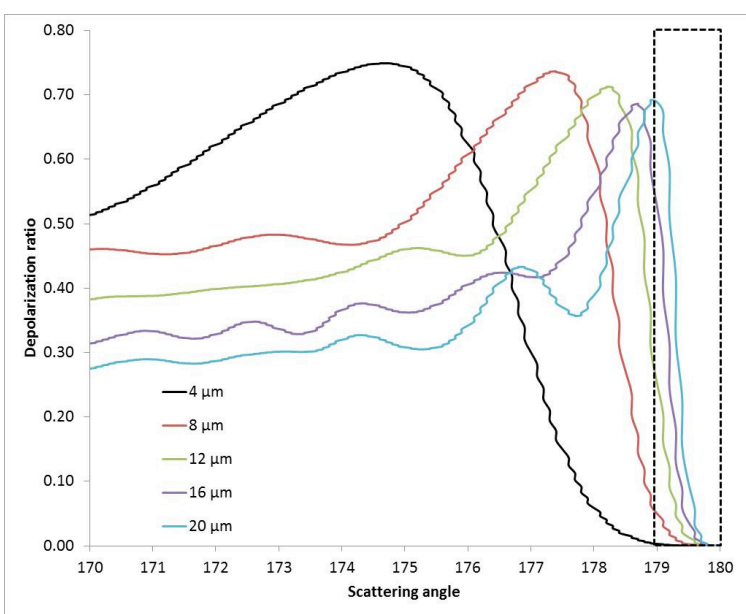

Figure 2. Depolarization ratios for water droplets of 4 , 8, 12, 16 and $20 \mu \mathrm{m}$ in diameter. The rectangular box represents a $1^{o}$ width.

\section{SCATTERING AND DEPOLARIZATION}

Double scattering events and higher scattering events leading to depolarization are illustrated in Figure 3. For clarity, only a single backscattering has been illustrated at distance $z_{c}$ from the lidar system. This backscattering is preceded by a forward scattering event that can come from different distances $z_{a}$. A lidar with a given FOV $\left(\theta_{i}\right)$ will measure the backscattered light without any distinction regarding the forward scattering event position in space. The second order scattering signal detected will be the sum of all those signals and it will be given by:

$$
P_{D}\left(z_{c}, \theta_{i}\right)=P\left(z_{c}\right) \int_{0}^{z_{c} \beta_{i}} \int_{0} F(z, \beta) \sin \beta \mathrm{d} \beta \mathrm{d} z,
$$

where $P\left(z_{c}\right)=P_{0} \exp \left[-2 \int_{0}^{z_{c}} \alpha(z) d z\right] \frac{c \tau}{2} \frac{4 \pi A}{z_{c}^{2}}$, and $F(z, \beta)=\left[\alpha_{s}(z) p(r, z, \beta)\right]\left[\alpha_{s}\left(z_{c}\right) p\left(r, z_{c}, \beta_{b}\right)\right]$, where, $c, \tau$ and $A$ are respectively the speed of light, the pulse width and the collecting optics area; $\alpha_{s}$ is the scattering extinction coefficient; $p(r, z, \beta)$ and $p\left(r, z_{c}, \beta_{b}\right)$ are the values of the phase function for the forward $(\beta)$ and backward scattering angles $\left(\beta_{b}=\pi-\beta+\theta\right)$ for a particle of radius $r ; z_{a}$ is the distance of the beginning of the cloud; $z_{c}$ is the distance where the scattered radiation is measured; the quantity $\left[\alpha_{s}(z) p(r, z, \beta)\right]$ represents the forward scattering coefficient while $\left[\alpha_{s}\left(z_{c}\right) p\left(r, z_{c}, \beta_{b}\right)\right]$ represents the backscattering coefficient. The FOV $\theta$ is easily related to the scattering angle $\beta$ in the simple geometric relation: $\tan \beta=\frac{z_{c} \tan \theta}{z_{c}-z}$. For compactness of the equations, the particle size dependence of the functions $F$ and $\delta_{\text {lin }}$ has been dropped out.

The depolarization ratio for scattering angle within $\beta_{i}$ is calculated as follows:

$$
\bar{\delta}_{\text {lin }}\left(z_{c}, \beta_{i}\right)=\frac{\int_{0}^{z_{c}} \int_{0}^{\beta_{i}} F(z, \beta) \delta_{l i n}\left(z_{c}, \beta_{b}\right) \sin \beta \mathrm{d} \beta \mathrm{d} z}{\int_{0}^{z_{c}} \int_{0_{i}}^{\beta_{i}} F(z, \beta) \sin \beta \mathrm{d} \beta \mathrm{d} z}
$$

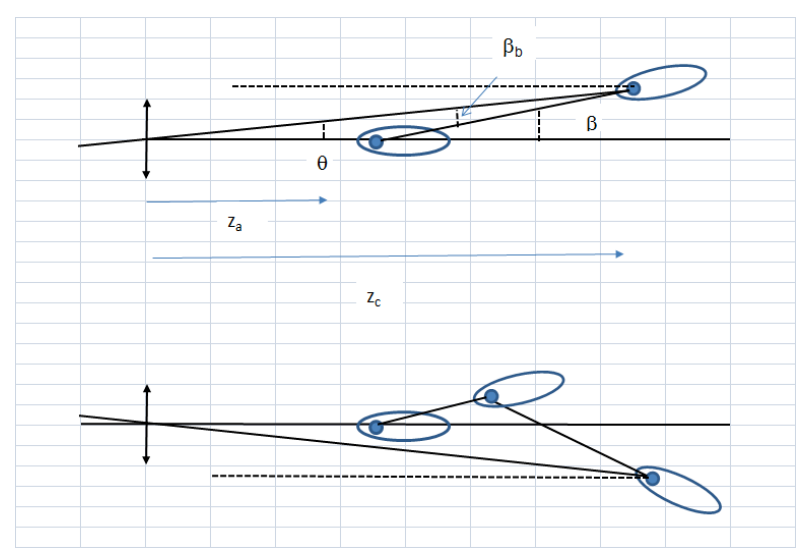

Figure 3. Double scattering processes and higher scattering processes leading to backscattering depolarization.

Figure 1 clearly shows that the depolarization ratios for scattering angles smaller than $20^{\circ}$ are very close to zero. Considering the small field of view covered by a typical lidar detector, the measured depolarization effect must come from the backscattering. Figure 4 shows the function $F(z, \beta) \delta_{\text {lin }}(z, \beta)$ as a function of the scattering angle $\beta$. 


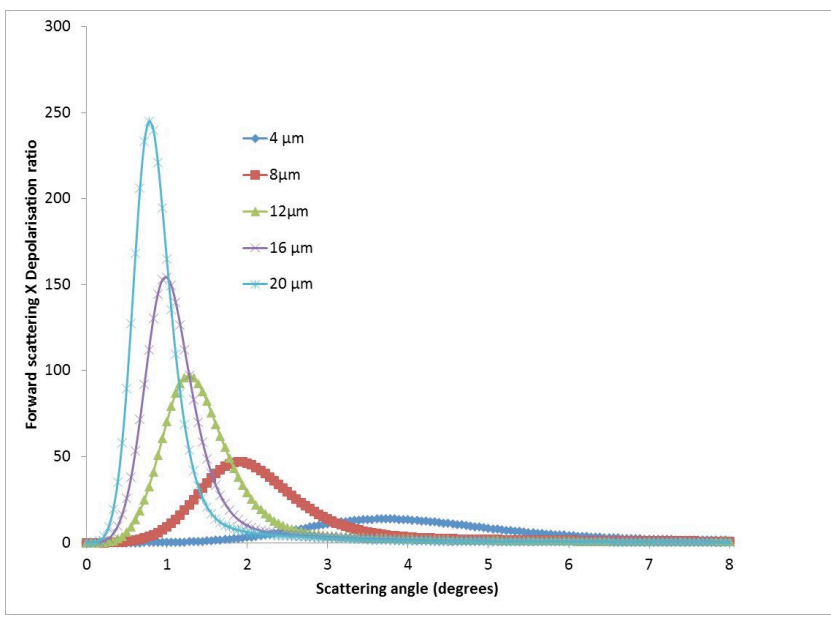

Figure 4. The product of the forward scattering phase function at scattering angle $\beta$ and the depolarization ratio at angle $\beta^{\prime}=\beta-\pi$ as a function of the scattering angle $\beta$.

Clearly the product of the forward scattering phase function at scattering angle $\beta$ and the depolarization ratio at angle $\beta^{\prime}=\beta-\pi$ as a function of scattering angle $\beta$ contains information on the size of the particle and a Multiple FOV lidar will be able to extract that information under some conditions. Can a single FOV depolarization measurement provide information on droplet size? Is there an optimal FOV to obtain information on droplet size?

In view of a better understanding of the problem, Figure 5 shows the product of normalized cumulative forward scattering and depolarization ratio as a function of scattering angle. The cumulative signal at a specific scattering angle is the signal which would be recorded by a lidar with that specific angle as FOV. The different arrows are for different cloud geometries and lidar FOVs. For small scattering angles, the average scattering angle, $\left\langle\beta>\right.$ over a penetration depth $\Delta z\left(\Delta z=z_{c}\right.$ $\left.z_{a}\right)$ is equal to: $\left\langle\beta>=\theta \frac{2 z_{c}+\Delta z}{\Delta z}\right.$.

From Fig. 5 and Table 1, it is clear that for average scattering angles greater than 2.5 degrees the product of cumulative forward scattering and depolarization ratio reach values very close to each other (except for the $4 \mu \mathrm{m}$ droplets). A small FOV would thus show better differentiation capability, which reinforces the interest of the depolarization single FOV lidar based method, but at the cost of an often significantly reduced signal.

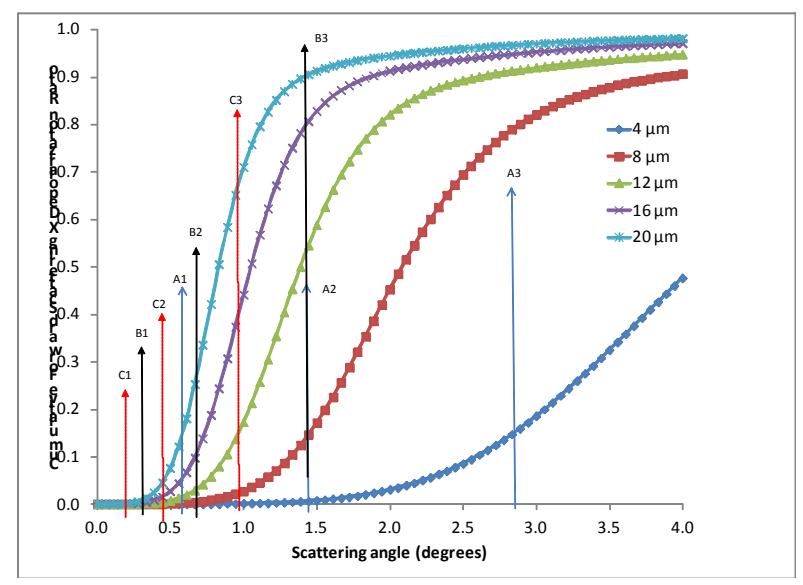

Figure 5. Normalized product of cumulative forward scattering and depolarization ratio as a function of scattering angle. The different identified arrows are for different cloud geometries and lidar FOVs.

Table 1 Average scattering angle within lidar FOV of 0.5 and 2 mrad for different cloud base and cloud penetration depth.

\begin{tabular}{|c|c|c|c|}
\hline & & $\begin{array}{l}\text { FOV: } 0.5 \\
\text { (mrad) }\end{array}$ & $\begin{array}{l}\text { FOV: } 2.0 \\
\text { (mrad) }\end{array}$ \\
\hline Cloud base $(\mathrm{m})$ & $\tau_{\mathrm{Z}(\mathrm{m})}$ & $\tau\left(\psi^{\mathrm{Deg}}\right)$ & $\tau \varepsilon($ Deg $)$ \\
\hline 500 & 10 & $0.72(\mathrm{~A} 1)$ & 2.89 \\
\hline 1000 & 10 & $1.44(\mathrm{~A} 2)$ & 5.76 \\
\hline 2000 & 10 & $2.87(\mathrm{~A} 3)$ & 11.49 \\
\hline 500 & 20 & 0.37 (B1) & 1.46 \\
\hline 1000 & 20 & 0.72 (B2) & 2.89 \\
\hline 2000 & 20 & 1.44 (B3) & 5.76 \\
\hline 500 & 30 & $0.25(\mathrm{C} 1)$ & 0.98 \\
\hline 1000 & 30 & $0.48(C 2)$ & 1.94 \\
\hline 2000 & 30 & $0.96(C 3)$ & 3.85 \\
\hline
\end{tabular}

So far we have focused our effort on understanding the physics of the depolarization effect caused by multiple scattering and its relation to the lidar FOV and did not address the effect of the cloud extinction profile. In the following and last section, the effect of cloud extinction profile will be studied using Monte Carlo simulation.

\section{MONTE CARLO SIMULATION}

The Undique Monte Carlo (MC) simulator is a multithread software based on the Bohren and Huffman Mie scattering computation routine. The simulator is made to reproduce the characteristics of an actual Flash Lidar system. It consists of an 
emitter/receiver system, a target and a propagation range including aerosols of various properties. The particularity of the Undique $\mathrm{MC}$ is its capability to image the scattered light on a detector array. See [6] for details.

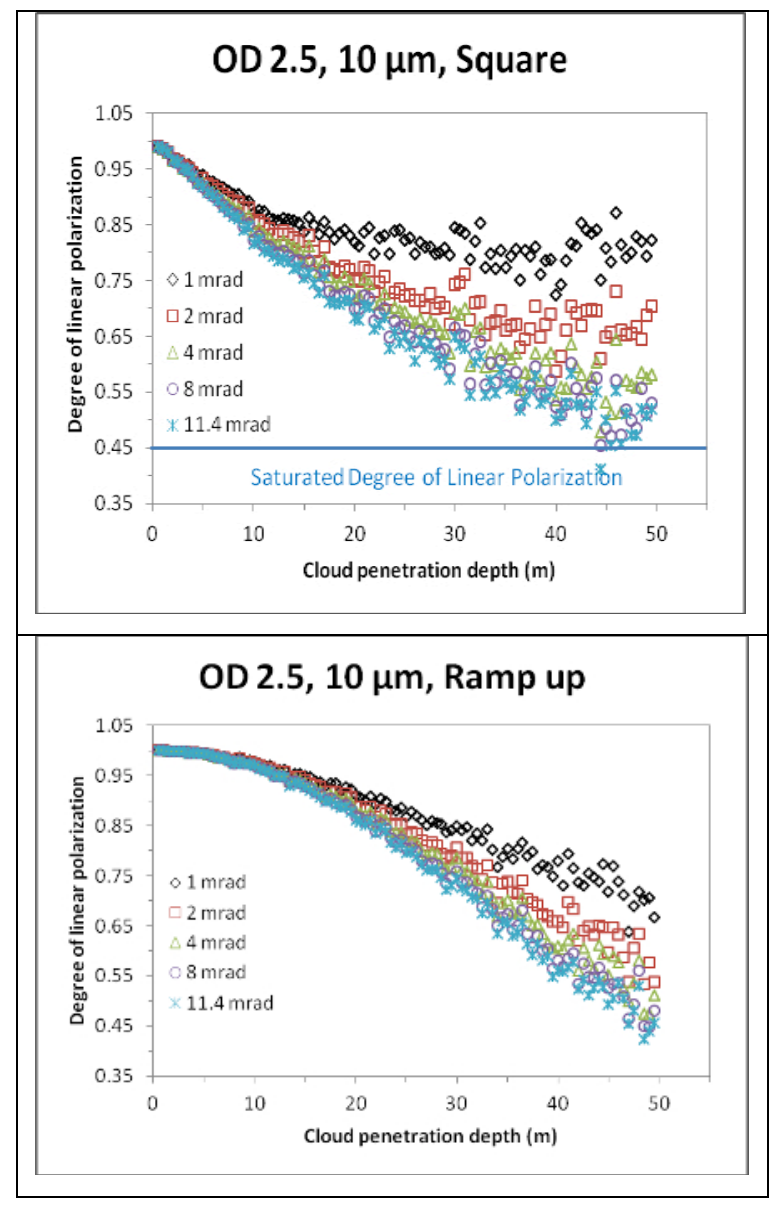

Figure 6 Degree of linear polarization as a function of cloud penetration depth.

The different parameters for the MC simulation are listed; Emitter: $532 \mathrm{~nm}, 0.5 \mathrm{mrad}$ beam divergence, Pulse width and shape: $10 \mathrm{~ns}$, Gaussian; Receiver: Receiver diameter and focal length; $0.2 \mathrm{~m}$ and $0.527 \mathrm{~m}$; Cloud base: $1000 \mathrm{~m}$, depth: $50 \mathrm{~m}$, optical depth: 2.5, profiles: Square and Ramp up.

Figure 6 shows the degree of linear polarization as a function of cloud penetration. Degree of linear polarization was used to facilitate comparison with [3]. The cloud extinction profile has a strong influence on the degree of linear polarization (or depolarization ratio) and such an effect is only partly accounted for in [3] and [4]. In absence of knowledge of cloud extinction profile or working hypothesis [4], the simple measurement of depolarization for a given FOV will most likely not be enough to provide information on droplet size.

\section{CONCLUSION}

Droplet size information is contained in the lidar depolarization signal, especially at small FOVs where the higher order scattering effects are less important and where the product of cumulative forward scattering and depolarization ratio shows distinct values as a function of droplet size for constant cloud extinction profile. A non-constant cloud profile will have an effect on the product of cumulative forward scattering and depolarization ratio and will most likely make droplet size recovery unreliable.

\section{REFERENCES}

[1] G. Roy, L. R. Bissonnette, C. Bastille, and G. Vallée, 1999: Retrieval of droplet-size density distribution from multiple field-of-view crosspolarized lidar signals, Appl. Opt. 38, pp.52025211.

[2] I. Veselovskii, M. Korenskii, V. Griaznov, D. N. Whiteman, M. McGill, G. Roy, L. Bissonnette "Information contain of data measured with multiple-field-of-view lidar," Appl. Opt. 45, 68396848(2006)

[3] K. Dukhyeon and J. Lee, 2014: Measuring cloud droplet effective radius and liquid water content using changes in degree of linear polarization along cloud depth, Opt. Lett., 39, pp. 3378-3381.

[4] D.P. Donovan, H. K. Baltink, , J.S. Henzing, S.R. de Roode and A.P. Siebesma, 2015,: A depolarization lidar-based method for the determination of liquid-cloud microphysical properties, Atmos. Meas. Tech.. 8, pp.237-266.

[5] K. Sassen, H. Zhao, 1995: Lidar multiple scattering in water droplet clouds: Toward an improved treatment, Opt. Rev., 2, pp. 394-400.

[6] G. Tremblay, G. Roy, 2015: High fidelity imaging algorithm for the Undique Monte Carlo simulator. 27rd International Laser Radar Conference, New York, USA. 